EDITORIAL

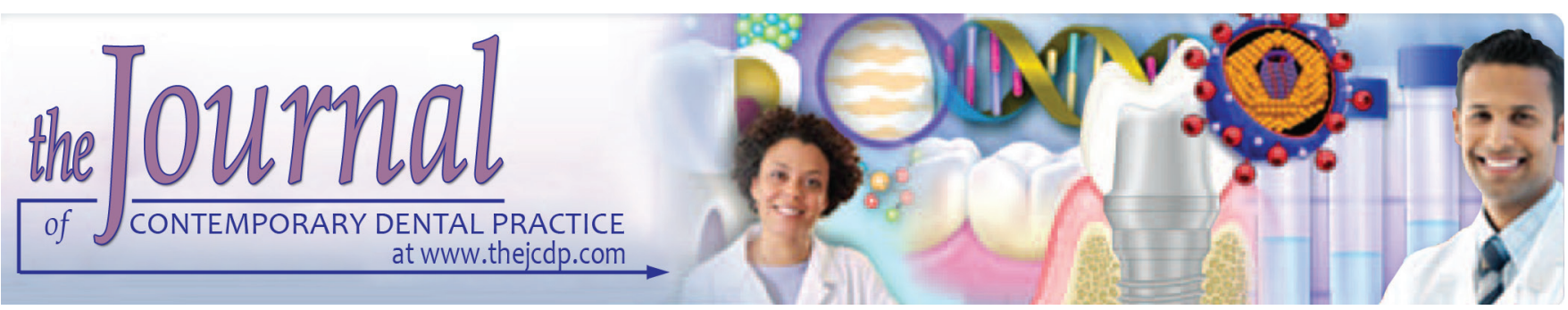

\title{
Effect on Quality of Life in Oral Cancer Patients after Radiation and Chemotherapy
}

\author{
${ }^{1}$ Quratul Ann Hussain, ${ }^{2}$ Kamran Habib Awan
}

How to cite this article: Hussain QA, Awan KH. Effect on Quality of Life in Oral Cancer Patients after Radiation and Chemotherapy. J Contemp Dent Pract 2016;17(2):97-98.

Almost $10 \%$ of the tumors that affect the human body are sited in the mouth. Oral cancer has the 6th highest occurrence rate among the diverse forms of malignancies. Excluding skin cancer, oral cancer is the most common form of cancer affecting the head and neck region. ${ }^{1}$ Although a number of adjunctive tools are available for the early detection of oral cancer, ${ }^{2,3}$ the survival rate is low. Low survival rates highlight the need for better care accessible to patients with oral cancer. The purpose of cancer therapy is to exclude or lessen tumor load. The outcome of the treatment depends on various variables, such as kinetics of tumor cell, effected site, and involvement of nearby vital structures. This helps in specifying a single or a multimodality therapy. ${ }^{4}$

Oral cancer treatment in the form of radiotherapy and chemotherapy compromises the oral cavity in various forms. This includes use of cytotoxic drugs and high-energy radiation that may result in both acute and long-term consequences to the oral mucosa and impaired bone marrow function. ${ }^{3}$ In addition, salivary glands, oral mucosa, mandibular musculature, teeth and alveolar bone are also affected. These changes occur both during and after completion of these treatment modalities. Oral epithelium becomes thin and ulcerated following chemotherapy and radiotherapy as proliferative ability of the oral epithelium is compromised. This damage can be either reversible or irreversible. ${ }^{5}$

\footnotetext{
1,2Department of Oral Medicine and Diagnostic Sciences College of Dentistry, King Saud University, Riyadh, Kingdom of Saudi Arabia

Corresponding Author: Kamran Habib Awan, Assistant Professor, Department of Oral Medicine and Diagnostic Sciences, College of Dentistry, King Saud University, Riyadh Kingdom of Saudi Arabia, Phone: (966)(1) 4677422, e-mail: kamranhabibawan@gmail.com
}

Tongue, cheeks, and soft palate are usually the primary sites affected. The initial lesion gradually enlarges leading to extreme pain and dysphagia. This leads to dehydration, a compromised nutritional status due to painful mastication, and a decreased quality of life. The cancer therapy not only affects the epithelium but also leads to poor cell proliferation and decreased turnover in connective tissue. Fibrosis is increased due to damage to fibroblasts and impairment of blood vessels leads to reduced vascularity and tissue ischemia. These changes decrease the competency of the tissues to heal and rebuild and fight infection.

Surgical management of intraoral lesions normally take in the primary lesion and the cervical lymph nodes and sometimes comorbidity at the expense of the spinal accessory nerve, internal jugular vein, or the sternocleidomastoid muscle which is usually present within a radical neck dissection or modified neck dissection. ${ }^{6}$ However, staging is crucial to decide whether surgery only is indicated or whether radiation or chemotherapy is also needed. The hazards and sequelae of surgery include cosmetic and functional disturbances and compromise of vascularity and nerve tissue. ${ }^{7}$

Risks and sequelae of radiation and chemotherapy include salivary gland pathoses, oral mucositis, rampant caries, demineralization, osteoradionecrosis, dysgeusia, and laryngeal alterations. Functional disabilities include impaired ability to eat, taste, swallow, and speak. There are also changes in taste perception of foods. High doses of radiation and chemotherapy affect development in children, resulting in alteration in tooth development and craniofacial growth. With chemotherapy, consequences are precisely related to the pharmacologic class of drug chosen as well as its dose and the extent of neutrophil depletion or leukopenia. ${ }^{7}$

Since cancer treatment can affect the oral tissues, a dentist should be aware of potential oral side effects. Forgoing oral disease can also complicate cancer management. So the proper role of a dentist in managing a cancer 
patient can benefit beyond the oral cavity. Oral health and quality of life of a patient are compromised in radiation and chemotherapy of the head and neck. Therapeutically essential oral care before, during, and after cancer treatment can prevent or ease the occurrence and severity of oral complications, improving equally patient survival and quality of life.

There is usually a short time period between diagnosis of cancer and start of treatment. If possible, a complete oral assessment should be done 1 month before cancer management starts. This provides suitable time for recovery in case any essential invasive dental procedure is required. The pretreatment assessment comprises of a detailed examination of hard and soft tissues, as well as suitable radiographs to identify likely causes of infection and pathology. Teeth with uncertain prognosis or directly associated with the tumor, or in the route of the radiation should be extracted well in time, to allow at least 3 weeks for healing time before radiotherapy. A dentist being a part of the palliative care team can provide psychological comfort, diagnosis, and maintenance of dental conditions including fulfillment of prosthetic requirements and directions for appropriate hydration of the oral mucosa.

\section{REFERENCES}

1. Chang TS, YC Su, Lee CC. Determinants for aggressive endof-life care for oral cancer patients. Medicine (Baltimore) 2015 Jan;94(4):e460.

2. Awan KH, Morgan PR, Warnakulasuriya S. Assessing the accuracy of autofluorescence, chemiluminescence and toluidine blue as diagnostic tools for oral potentially malignant disorders-a clinicopathological evaluation. Clin Oral Investig 2015 Dec;19(9):2267-2272.

3. Awan KH, Patil S. Efficacy of autofluorescence imaging as an adjunctive technique for examination and detection of oral potentially malignant disorders: a systematic review. J Contemp Dent Pract 2015 Sep 1:16(9):744-749.

4. Kolokythas A. Long-term surgical complications in the oral cancer patient: a comprehensive review. Part I. J Oral Maxillofac Res 2010 Jul-Sep;1(3):e1.

5. Patel Y, Bahlhorn H, Zafar S, Zwetchkenbaum S, Eisbruch A, Murdoch-Kinch CA. Survey of Michigan dentists and radiation oncologists on oral care of patients undergoing head and neck radiation therapy. J Mich Dent Assoc 2012 Jul;94(7):34-45.

6. Thanvi L, Bumb D. Impact of dental considerations on the quality of life of oral cancer patients. Indian J Med Pediatr Oncol 2014 Jan-Mar;35(1):66-70.

7. Vikas K, Shalini B, Amit P. Chemotherapy and oral complications - the most neglected side of cancer. J Adv Med Dent Sci Res 2015 Jan-Mar;3(1):71-80. 